\title{
Strong fragmentation and coagulation with power-law rates
}

\author{
Jacek Banasiak • Wilson Lamb • \\ Matthias Langer
}

Received: 13 April 2012 / Accepted: 22 October 2012 / Published online: 8 January 2013

(C) The Author(s) 2013. This article is published with open access at Springerlink.com

\begin{abstract}
Existence of global classical solutions to fragmentation and coagulation equations with unbounded coagulation rates has been recently proved for initial conditions with finite higher-order moments. These results cannot be directly generalized to the most natural space of solutions with finite mass and number of particles due to the lack of precise characterization of the domain of the generator of the fragmentation semigroup. In this paper we show that such a generalization is possible in the case when fragmentation is described by power-law rates, which are commonly used in engineering practice. This is achieved through direct estimates of the resolvent of the fragmentation operator, which in this case is explicitly known, proving that it is sectorial and carefully intertwining the corresponding intermediate spaces with appropriate weighted $L_{1}$ spaces.
\end{abstract}

Keywords Analytic semigroup - Fractional power of operators · Fragmentation-coagulation equation · Real interpolation $\cdot$ Semilinear Cauchy problem

\section{Introduction}

Coagulation and fragmentation processes can be found in many important areas of science and engineering. Examples range from astrophysics [1], blood clotting [2], colloidal chemistry and polymer science [3,4] to molecular beam epitaxy [5]. An efficient way of modelling the dynamical behaviour of such processes is to use a rate equation

This paper is dedicated to Professor P. G. L. Leach on his 70th birthday.

J. Banasiak $(\bowtie)$

School of Mathematics, Statistics and Computer Sciences, University of KwaZulu-Natal, Durban, South Africa

e-mail: banasiak@ukzn.ac.za

J. Banasiak

Institute of Mathematics, Technical University of Łódź, Łódź, Poland

e-mail: jacek.banasiak@p.lodz.pl

W. Lamb · M. Langer

Department of Mathematics and Statistics, University of Strathclyde, Glasgow, UK

e-mail:w.lamb@strath.ac.uk

M. Langer

e-mail: m.langer@strath.ac.uk 
which describes the evolution of the distribution of interacting clusters or particles with respect to their size or mass. If it is assumed that the particle-size variable is real and positive, and the particle-size distribution varies only as a result of coalescence and fragmentation, with reaction rates that depend on the sizes of the individual particles involved, then we arrive at the integro-differential equation

$$
\begin{aligned}
\partial_{t} u(x, t)= & -a(x) u(x, t)+\int_{x}^{\infty} a(y) b(x \mid y) u(y, t) \mathrm{d} y-u(x, t) \int_{0}^{\infty} k(x, y) u(y, t) \mathrm{d} y \\
& +\frac{1}{2} \int_{0}^{x} k(x-y, y) u(x-y, t) u(y, t) \mathrm{d} y .
\end{aligned}
$$

Equation (1.1) is usually referred to as the continuous coagulation-fragmentation equation. The function $u$ is interpreted as a density function, with $u(x, t)$ denoting the density of particles of size $x$ at time $t$. The coagulation kernel $k(x, y)$ then represents the rate at which particles of size $x$ coalesce with particles of size $y$. The fragmentation terms have a similar interpretation, with $a(x)$ representing the overall rate of fragmentation of an $x$-sized particle while $b(x \mid y)$ gives the average number of particles of size $x$ produced when a particle of size $y>x$ breaks up. For the total mass in the system to remain constant during fragmentation, $b$ must satisfy the condition

$$
\int_{0}^{y} x b(x \mid y) \mathrm{d} x=y, \quad y \in \mathbb{R}_{+} .
$$

A more detailed explanation of each of the terms in Eq. (1.1) can be found in [6-8].

In many physical problems, it has been found that appropriate forms for $a$ and $b$ are given by the power-law formulae

$$
a(x)=x^{\alpha} \text { and } b(x \mid y)=(v+2) \frac{x^{\nu}}{y^{\nu+1}},
$$

where $\alpha$ and $v$ are real parameters which we will specify later; see [3] and the references contained therein. An additional reason for such a choice of $a$ and $b$ is that the resulting fragmentation equation, obtained from (1.1) by setting $k=0$, can be explicitly solved, though the formulae can be rather involved. Thus, in the current paper, we shall analyse

$$
\begin{aligned}
\partial_{t} u(x, t)= & -x^{\alpha} u(x, t)+(v+2) x^{\nu} \int_{x}^{\infty} y^{\alpha-v-1} u(y, t) \mathrm{d} y \\
& -u(x, t) \int_{0}^{\infty} k(x, y) u(y, t) \mathrm{d} y+\frac{1}{2} \int_{0}^{x} k(x-y, y) u(x-y, t) u(y, t) \mathrm{d} y .
\end{aligned}
$$

It is easy to see that the aforementioned local law of mass conservation (1.2) is satisfied, provided that $v>-2$, and the expected number of particles resulting from the fragmentation of a size- $y$ parent is given by

$n_{0}(y):=\int_{0}^{y} b(x \mid y) \mathrm{d} x= \begin{cases}\frac{v+2}{v+1} & \text { if } v>-1, \\ \infty & \text { if } v \leq-1 .\end{cases}$

It can also be proved that, for the fragmentation process modelled in (1.3) to be physically correct, we must have $v \leq 0$; see [9, Sect. 8.2] and [6]. Thus we assume throughout this paper that $-1<v \leq 0$.

Turning to the coagulation kernel $k$, we assume that it is a measurable symmetric function such that, for some $K>0$ and $0 \leq \sigma \leq \rho<\alpha$,

$0 \leq k(x, y) \leq K\left((1+x)^{\rho}(1+y)^{\sigma}+(1+x)^{\sigma}(1+y)^{\rho}\right)$ 
for $x, y \in \mathbb{R}_{+}$. This will suffice to show local in time solvability of (1.3), whereas to show that the solutions are global in time we need to strengthen (1.5) to

$0 \leq k(x, y) \leq K\left((1+x)^{\rho}+(1+y)^{\rho}\right)$

for $x, y \in \mathbb{R}_{+}$and $0 \leq \rho \leq 1, \rho<\alpha$.

In fragmentation and coagulation problems, two spaces are most often used due to their physical relevance. In the space $Y=L_{1}\left(\mathbb{R}_{+}, x \mathrm{~d} x\right)$ the norm of a non-negative element $f$, given by $\int_{0}^{\infty} f(x) x \mathrm{~d} x$, represents the total mass of the system, whereas the norm of a non-negative element $f$ in the space $X_{0}=L_{1}\left(\mathbb{R}_{+}, \mathrm{d} x\right), \int_{0}^{\infty} f(x) \mathrm{d} x$, gives the total number of particles in the system.

It is well known that the fragmentation equation, with a fragmentation rate $a$ that is unbounded as $x \rightarrow \infty$, has good properties in $Y$ but is ill posed in $X_{0}$; see [10]. On the other hand, the coagulation operator behaves well in $X_{0}$ and in $L_{1}\left(\mathbb{R}_{+},(1+x) \mathrm{d} x\right)$ but not in $Y$ alone. Note that this is one of the reasons for the restriction $v \in(-1,0]$, though the fragmentation equation itself is well posed in $Y$ also for $v \in(-2,-1]$, in which case the number of particles created in each fragmentation event is considered to be infinite.

We use the scale of spaces with finite higher moments

$X_{m}=L_{1}\left(\mathbb{R}_{+}, \mathrm{d} x\right) \cap L_{1}\left(\mathbb{R}_{+}, x^{m} \mathrm{~d} x\right)=L_{1}\left(\mathbb{R}_{+},\left(1+x^{m}\right) \mathrm{d} x\right)$,

where $m \in \mathbb{M}:=[1, \infty)$. We extend this definition to $X_{0}=L_{1}\left(\mathbb{R}_{+}, \mathrm{d} x\right)$. The natural norm in $X_{m}$ is denoted by $\|\cdot\|_{m}$. Recall also that the space $L_{1}\left(\mathbb{R}_{+}, x \mathrm{~d} x\right)$ is denoted by $Y$.

Though our main interest in this paper is the space $X_{1}$, we shall make frequent use of results that can be shown to hold in $X_{m}$, where $m>1$ when $0<\alpha \leq 1$, and $m \geq \alpha$ when $\alpha>1$. We note that the continuous injection $X_{m} \hookrightarrow X_{1}, m>1$, means that any solution in $X_{m}$ is also a solution in the basic space $X_{1}$. Also, due to the nature of the problem, our analysis is carried out in $X_{m,+}$, where for any partially ordered space $Z, Z_{+}$denotes the positive cone of $Z$.

Previous rigorous investigations into coagulation-fragmentation equations have relied primarily on two different methods. One strategy, used, for example, in [11-14], establishes the existence of weak solutions by means of weak compactness methods. The uniqueness of such solutions then requires additional assumptions and separate techniques. Although this strategy works very well with pure coagulation problems, for the full coagulation-fragmentation equation it requires that the fragmentation part be in some sense subordinated to the coagulation kernel; see [15]. An alternative approach, introduced in [16] and further developed in [7,8,15,17,18], treats (1.1) as a Lipschitz perturbation of the linear fragmentation problem. Here, powerful methods from substochastic semigroup theory [9, Chapter 6] enable a wide range of unbounded fragmentation kernels to be catered for, but then the coagulation process must, in a certain way, be controlled by fragmentation.

As we mentioned above, one of the problems related to the analysis of the combined coagulation-fragmentation equation is that it must be considered in the space $X_{1}$ in which both the total number and total mass of clusters are controlled. While the natural space for fragmentation is $L_{1}\left(\mathbb{R}_{+}, x \mathrm{~d} x\right)$, for coagulation it is easier to work in $L_{1}\left(\mathbb{R}_{+}, \mathrm{d} x\right)$. To work in the combination of these two spaces one has to impose additional assumptions on the fragmentation and coagulation rates. In particular, in $[8,17,19]$, it was assumed that the fragmentation rate $a$ is bounded by a linear function while the coagulation rate was either constant or bounded. Significant progress was made when it was recognised that both fragmentation and coagulation operators have better properties in the spaces $X_{m}$ of functions with finite higher moments. This enabled the sublinearity assumption on $a$ to be removed in [15], but with the boundedness assumption on $k$ still required. A further step was made with the realization that the fragmentation operator is sectorial in $X_{m}$ for sufficiently large $m$ and for a large class of particle-size distribution functions $b$. An immediate and beneficial consequence of this is that intermediate spaces associated with the fragmentation operator can be introduced and the theory developed in [20, Chapter 2] can then be applied to establish the well-posedness in such spaces of the semilinear problem (1.1). Using this approach, we proved the existence of global solutions in $X_{m}$ for a large class of physically relevant unbounded coagulation kernels, such as the shear kernel [21,22], or the modified Smoluchowski kernel, [23]; see [24,25].

Unfortunately, the estimates allowing one to prove that the fragmentation operator is sectorial in $X_{m}, m>1$ break down for $m=1$. While the solutions to (1.3) in $X_{m}$ are also solutions in $X_{1}$, they are only available for a 
restricted class of initial conditions, since the value of $m$ can be very large, depending on the rate of growth of $a$ and $b$.

Another stumbling block in the analysis of (1.1) was that there had been no results on semigroup generation in $X_{1}$ for fast growing fragmentation rates. This drawback was resolved in [26] for the case when $b(x \mid y)=\beta(x) \gamma(y)$. This separability assumption played a key role in the analysis carried out in [26] as it meant that an explicit representation could be obtained for the resolvent of the fragmentation operator. Clearly, this separability condition is satisfied for the class of so-called power-law fragmentation processes that we consider in this paper. The fact that an explicit resolvent formula is therefore available for these physically relevant fragmentation processes enables us to show that the intermediate space approach used in $[24,25]$ within the framework of higher moment spaces is also applicable in the space $X_{1}$.

We shall also discuss an interesting consequence of the sectoriality of the fragmentation operator, namely the existence of 'spurious' solutions to fragmentation equations, that is, solutions which, for $t>0$, live in the observable, physical, space $X_{1}$ but which originate from non-physical initial mass distributions. The example used to illustrate this phenomenon in Sect. 6 is a continuous counterpart of a similar solution to a discrete fragmentation equation which was constructed in [27] (without realizing that it is non-physical) and explained in [28].

\section{Formulation of main results}

In [25], we were able to establish several results, including analyticity, relating to the semigroup associated with the fragmentation part of Eq. (1.1). These results were proved under certain assumptions on the fragmentation kernels $a$ and $b$, and it was noted that the underlying analysis could not be applied in the space $X_{1}$, but instead required a higher moment space $X_{m}$ with the value of $m$ being dependent on $a$ and $b$. It is a routine matter to show that the assumptions required for [25, Theorem 2.1] to hold are satisfied for the power-law rate kernels $a$ and $b$ that we consider here. Clearly,

$$
\begin{aligned}
& a(x)=x^{\alpha} \leq a_{0}\left(1+x^{j}\right) \quad \text { with } a_{0}=1, j=\alpha, \\
& n_{0}(x) \leq b_{0}\left(1+x^{l}\right) \quad \text { with } \quad b_{0}=\frac{v+2}{v+1}, l=0,
\end{aligned}
$$

by (1.4), and

$$
\liminf _{y \rightarrow \infty} \frac{y^{m}-\int_{0}^{y} b(x \mid y) x^{m} \mathrm{~d} x}{y^{m}}=\frac{m-1}{v+m+1} \in(0, \infty)
$$

with $m$ chosen as above; see Eqs. (5) and (12) in [25]. It follows immediately that the results on the analyticity of the fragmentation semigroup that are stated in [25, Theorem 2.1] are also valid for the power-law case, under the assumptions that

$\alpha \geq 0, \quad-1<v \leq 0$.

For completeness, and to enable comparison with the new results that we prove in this paper, it is useful to summarise the key points that can be deduced directly from [25]. First let us introduce the expression

$$
\begin{aligned}
(\mathcal{F} f)(x) & =-x^{\alpha} f(x)+(\nu+2) x^{v} \int_{x}^{\infty} y^{\alpha-v-1} f(y) \mathrm{d} y, \quad x \in \mathbb{R}_{+}, \\
& =:-(\mathcal{A} f)(x)+(\mathcal{B} f)(x) .
\end{aligned}
$$

We assume that $m \geq \alpha$ if $\alpha>1$ or $m>1$ if $0<\alpha \leq 1$. Note that we do not consider $\alpha=0$ in the following, as in this case the fragmentation operator is bounded and so can be dealt with in a far more routine manner. For any $m \in \mathbb{M}$, we define $\left(A_{m} f\right)(x):=\mathcal{A} f(x)=x^{\alpha} f(x)$ on

$$
D\left(A_{m}\right)=\left\{f \in X_{m}: \mathcal{A} f \in X_{m}\right\}=X_{m+\alpha}
$$

and let $B_{m}$ be the restriction to $D\left(A_{m}\right)$ of $\mathcal{B}$. We denote by $F_{m}=-A_{m}+B_{m}$ the fragmentation operator in $X_{m}$ with domain $D\left(A_{m}\right)$. Most statements of the following theorem are from [25, Theorem 2.1]. 
Theorem 2.1 Let $\alpha>0$ and $v \in(-1,0]$. Then the operator $F_{m}$, with $m$ as above, generates a positive, analytic and quasicontractive semigroup $\left(S_{F_{m}}(t)\right)_{t \geq 0}$ in $X_{m}$ of type not exceeding $1 /(v+1)$.

The only part of the above result that requires any explanation is the growth constant, as [25, Theorem 2.1 (i)] can be used only to deduce that the growth constant is at most $4 a_{0} b_{0}=4(v+2) /(v+1)$. However, the calculations leading to [25, Eq. (20)] show that, in this case, $1 /(v+1)$ is an upper bound for the rate constant.

The results on the combined coagulation-fragmentation equation that are proved in [25, Theorems 2.2 and 2.3] can also be applied to the initial-value problem (1.3). Here we note that the assumptions (1.5) and (1.6) that are imposed on the coagulation kernel $k$ take a slightly different form from those stated in [25, Eqs. $(6,7)]$. However, the conditions we use here can be seen to be equivalent to the latter on replacing $\alpha$ and $\beta$ in [25] by $\rho \alpha$ and $\rho \gamma$, respectively. Moreover, the intermediate space $X_{m}^{(\alpha)}$ that features in [25, Eq. (15)] then becomes $X_{m+\rho}$. Consequently, if we introduce a nonlinear operator $C_{m}$ in $X_{m}$ defined for $f$ from a suitable subset of $X_{m}$ by the formula

$\left(C_{m} f\right)(x):=-f(x) \int_{0}^{\infty} k(x, y) f(y) \mathrm{d} y+\frac{1}{2} \int_{0}^{x} k(x-y, y) f(x-y) f(y) \mathrm{d} y$,

then the initial-value problem for (1.3) can be written as an abstract semilinear Cauchy problem in $X_{m}$

$u_{t}=-A_{m} u+B_{m} u+C_{m} u, \quad u(0)=\stackrel{\circ}{u}$,

where $u_{t}$ denotes the strong $X_{m}$ derivative of $u$. Theorems 2.2 and 2.3 in [25] lead immediately to the following theorem.

Theorem 2.2 Let $\alpha, v$ and $k$ satisfy the conditions (2.3) and (1.5) with $0 \leq \sigma \leq \rho<\alpha$, and let $m \geq \alpha$ if $\alpha>1$ and $m>1$ if $0<\alpha \leq 1$. Then,

(i) for each $\stackrel{\leftrightarrow}{u} \in X_{m+\rho,+}$, there exists $\tau>0$ such that (2.4) has a unique non-negative classical solution $u \in C\left([0, \tau), X_{m+\rho}\right) \cap C^{1}\left((0, \tau), X_{m}\right) \cap C\left((0, \tau), D\left(A_{m}\right)\right)$;

(ii) any local solution from (i) is global in time if, additionally, $k$ satisfies (1.6).

The situation changes, however, for $m=1$. First, it is known [9, Subsection 8.3.1] that there is a positive contractive semigroup $\left(S_{F}(t)\right)_{t \geq 0}$ on $Y$ generated by the closure $(F, D(F))$ of $\mathcal{F}$ restricted to $\{f \in Y: \mathcal{A} f \in Y\}$. Further, by [26], there is a positive semigroup $\left(S_{F_{1}}(t)\right)_{t \geq 0}$ on $X_{1}$ generated by the closure $\left(F_{1}, D\left(F_{1}\right)\right)$ of $-A_{1}+B_{1}$, but apart from the specific case when $\alpha=1$ and $v=0$, which we examine in some detail in Sect. 6, no explicit characterization of either $D(F)$ or $D\left(F_{1}\right)$ has yet been found. Nevertheless, for the general power-law case we can prove the following theorem.

Theorem 2.3 Let $\alpha>0$ and $v \in(-1,0]$. Then the semigroups $\left(S_{F}(t)\right)_{t \geq 0}$ and $\left(S_{F_{1}}(t)\right)_{t \geq 0}$ are analytic in, respectively, $Y$ and $X_{1}$.

The proof of Theorem 2.2 was facilitated by the fact that intermediate spaces associated with $F_{m}$ could be identified, via real interpolation between $D\left(F_{m}\right)=D\left(A_{m}\right)=X_{m+\rho}$ and $X_{m}$, with weighted spaces $X_{m+\theta \rho}, 0<\theta<1$. Unfortunately, due to $D\left(F_{1}\right) \neq D\left(A_{1}\right)$, such an identification is no longer feasible for $m=1$. We shall still use intermediate spaces associated with $F_{1}$, but they no longer have a Banach lattice structure and we will have to sandwich them between spaces of type $X_{1+\xi}$ with different $\xi$ s, which makes the final results slightly different from those in Theorem 2.2. We prove the following theorem.

Theorem 2.4 Let $\alpha>0$ and $v \in(-1,0]$, and let (1.5) with $0 \leq \sigma \leq \rho<\alpha$ hold. Then, for any $\beta>\rho$ and $\stackrel{\circ}{u} \in X_{1+\beta,+}$, there is $\tau>0$ such that the problem (1.3) has a unique non-negative solution $u$ that satisfies $u \in C\left([0, \tau), X_{1+\rho}\right) \cap C^{1}\left((0, \tau), X_{1}\right) \cap C\left((0, \tau), D\left(F_{1}\right)\right)$. If, additionally, (1.6) is satisfied, then this solution is global in time. 


\section{Linear part: proof of Theorem 2.3}

We examine the semigroups $\left(S_{F_{1}}(t)\right)_{t \geq 0}$ and $\left(S_{F}(t)\right)_{t \geq 0}$ generated in $X_{1}$ and $Y$, respectively, by the fragmentation operators $F_{1}$ and $F$. Our aim is to establish that each semigroup is analytic. To this end, we use [20, Proposition 2.1.11], which states the following: If, for some $\omega \in \mathbb{R}$, the half-plane $\{z \in \mathbb{C}: \operatorname{Re} z \geq \omega\}$ belongs to the resolvent set of a closed operator $T$ and

$\|R(\lambda)\| \leq \frac{M}{|\lambda|}, \quad \lambda \in \mathbb{C}, \operatorname{Re} \lambda \geq \omega$,

with some $M>0$ and where $R(\lambda)$ denotes the resolvent of $T$, then $T$ is sectorial and hence generates an analytic semigroup.

To obtain a formula for the resolvent $R(\lambda)$ of $F_{1}$ and $F$ we use a result from [26], where the more general fragmentation equation

$\partial_{t} u(x, t)=-a(x) u(x, t)+\int_{x}^{\infty} a(y) \beta(x) \gamma(y) u(y, t) \mathrm{d} y$

was investigated, where $a, \beta, \gamma$ are real-valued functions. Under the assumption (see [26, Corollary 2 and formula (36)]) that, for $\lambda \in \mathbb{C}, \operatorname{Re} \lambda>0$,

$\lim _{x \rightarrow 0^{+}} a(x) \in[0, \infty]$,

$\lim _{x \rightarrow 0^{+}}\left(\lambda x B_{\lambda}(x)\right) \in(1, \infty)$

where $\quad B_{\lambda}=\frac{\beta(x) \gamma(x)}{\lambda+a(x)}$ and $x B_{\lambda}(x)$ is Hölder continuous at $x=0$,

it can be established (see [26, Theorem 2 and Corollary 3]) that the resolvent of the generator of the associated semigroups in $X_{1}$ and $Y$ (denoted by $X_{0,1}$ and $X_{1}$ in [26]) is given by

$[R(\lambda) f](x)=\frac{f(x)}{\lambda+a(x)}+\frac{B_{\lambda}(x)}{x} \mathrm{e}^{\lambda \int_{1}^{x} B_{\lambda}(s) \mathrm{d} s} \int_{x}^{\infty} \frac{s a(s) f(s)}{\lambda+a(s)} \mathrm{e}^{-\lambda \int_{1}^{s} B_{\lambda}(r) \mathrm{d} r} \mathrm{~d} s$.

In our case $a(x)=x^{\alpha}(\alpha>0)$ and $B_{\lambda}(x)=(\nu+2) /\left(x\left(\lambda+x^{\alpha}\right)\right)$. Therefore, conditions (3.1)-(3.3) are satisfied. Moreover,

$$
\begin{aligned}
\lambda \int_{1}^{x} B_{\lambda}(s) \mathrm{d} s & =(v+2) \int_{1}^{x}\left(\frac{1}{s}-\frac{s^{\alpha-1}}{\lambda+s^{\alpha}}\right) \mathrm{d} s=\frac{\nu+2}{\alpha}\left(\alpha \ln x-\ln \left(\lambda+x^{\alpha}\right)+\ln (\lambda+1)\right) \\
& =\ln \left(\left(\frac{(\lambda+1) x^{\alpha}}{\lambda+x^{\alpha}}\right)^{(\nu+2) / \alpha}\right),
\end{aligned}
$$

and, on substituting into (3.4), we can conclude that the resolvents of $F_{1}$ and $F$ are given by the expression

$[R(\lambda) f](x)=\frac{f(x)}{\lambda+x^{\alpha}}+\frac{(\nu+2) x^{\nu}}{\left(\lambda+x^{\alpha}\right)^{\omega+1}} \int_{x}^{\infty}\left(\lambda+s^{\alpha}\right)^{\omega-1} s^{\alpha-\nu-1} f(s) \mathrm{d} s, \quad \operatorname{Re} \lambda>0$,

where

$\omega:=\frac{\nu+2}{\alpha} \in\left(\frac{1}{\alpha}, \frac{2}{\alpha}\right)$.

In [26] the formula was shown only for real positive $\lambda$, but one can see from the proof that the formula is still valid for non-real $\lambda$ in the right half-plane if fractional powers are defined with a cut along the negative half-line.

We need several estimates, which will be established in the following lemmas. 
Lemma 3.1 For each $p>0$, there exist positive constants $c_{p} \leq 1$ and $c_{p}^{\prime} \geq 1$ such that

$c_{p}\left(a^{p}+b^{p}\right) \leq(a+b)^{p} \leq c_{p}^{\prime}\left(a^{p}+b^{p}\right) \quad$ for all $a, b \geq 0$.

Proof Let $0<q \leq r$. Then, for all $a, b \geq 0$,

$\left(\frac{a^{q}+b^{q}}{2}\right)^{1 / q} \leq\left(\frac{a^{r}+b^{r}}{2}\right)^{1 / r}$ and $\left(a^{q}+b^{q}\right)^{1 / q} \geq\left(a^{r}+b^{r}\right)^{1 / r}$;

see, e.g. [29, (2.9.1) and (2.10.3)]. Combining the inequalities in (3.8) with either $q=1$ or $r=1$ then leads directly to (3.7).

Lemma 3.2 For every $\gamma>0$, there exist positive constants $C_{\gamma} \leq 1$ and $C_{\gamma}^{\prime} \geq 1$ such that

$C_{\gamma}\left(|\lambda|^{\gamma}+x^{\gamma}\right) \leq|\lambda+x|^{\gamma} \leq C_{\gamma}^{\prime}\left(|\lambda|^{\gamma}+x^{\gamma}\right)$

for every $\lambda \in \mathbb{C}$, with $\operatorname{Re} \lambda \geq 0$, and every $x \in[0, \infty)$.

Proof For $\lambda=\mu+\mathrm{i} v, \mu \geq 0, v \in \mathbb{R}, x \geq 0$, we have, by (3.7),

$$
\begin{aligned}
|\lambda+x|^{\gamma} & =\left((\mu+x)^{2}+v^{2}\right)^{\frac{\gamma}{2}} \leq c_{\frac{\gamma}{2}}^{\prime}\left((\mu+x)^{\gamma}+|\nu|^{\gamma}\right) \\
& \leq c_{\frac{\gamma}{2}}^{\prime} c_{\gamma}^{\prime}\left(\mu^{\gamma}+x^{\gamma}+|\nu|^{\gamma}\right) \leq \frac{c_{\frac{\gamma}{2}}^{\prime} c_{\gamma}^{\prime}}{c \frac{\gamma}{2}}\left(\left(\mu^{2}+|\nu|^{2}\right)^{\frac{\gamma}{2}}+x^{\gamma}\right)=C_{\gamma}^{\prime}\left(|\lambda|^{\gamma}+x^{\gamma}\right) .
\end{aligned}
$$

The other inequality is proved in a similar way.

Lemma 3.3 Let $\alpha>0, v \in(-1,0], \gamma>-1$ and let $\omega$ be as in (3.6). Then,

(a) there exists a positive constant $C_{\alpha, v}$ such that

$$
\int_{0}^{s} \frac{x^{\gamma}}{\left|\lambda+x^{\alpha}\right|^{\omega+1}} \mathrm{~d} x \leq \frac{C_{\alpha, \nu}}{|\lambda|^{1+\frac{v+1-\gamma}{\alpha}}} \int_{0}^{s /|\lambda|^{1 / \alpha}} \frac{t^{\gamma}}{\left(1+t^{\alpha}\right)^{\omega+1}} \mathrm{~d} t
$$

for all $s>0$ and $\lambda \in \mathbb{C}$ with $\operatorname{Re} \lambda>0$;

(b) under the additional assumption that $\gamma<\alpha+v+1$, there exists a positive constant $M_{\alpha, v, \gamma}$ such that

$$
\int_{0}^{s} \frac{x^{\gamma}}{\left|\lambda+x^{\alpha}\right|^{\omega+1}} \mathrm{~d} x \leq \frac{M_{\alpha, \nu, \gamma}}{|\lambda|^{1+\frac{v+1-\gamma}{\alpha}}}
$$

$$
\text { for all } s>0 \text { and } \lambda \in \mathbb{C} \text { with } \operatorname{Re} \lambda>0 \text {. }
$$

Proof (a) On setting $C_{\alpha, v}:=1 / C_{1}^{\omega+1}$ and using (3.9) we obtain

$$
\int_{0}^{s} \frac{x^{\gamma}}{\left|\lambda+x^{\alpha}\right|^{\omega+1}} \mathrm{~d} x \leq C_{\alpha, \nu} \int_{0}^{s} \frac{x^{\gamma}}{\left(|\lambda|+x^{\alpha}\right)^{\omega+1}} \mathrm{~d} x
$$

and (3.10) follows in a routine manner with the substitution $x=|\lambda|^{1 / \alpha} t$.

(b) The inequality in (3.10) leads to

$$
\int_{0}^{s} \frac{x^{\gamma}}{\left|\lambda+x^{\alpha}\right|^{\omega+1}} \mathrm{~d} x \leq \frac{C_{\alpha, \nu}}{|\lambda|^{1+\frac{v+1-\gamma}{\alpha}}} \int_{0}^{\infty} \frac{t^{\gamma}}{\left(1+t^{\alpha}\right)^{\omega+1}} \mathrm{~d} t .
$$

As the additional condition on $\gamma$ implies that $\alpha(\omega+1)-\gamma=\alpha+\nu+2-\gamma>1$, the integral on the right-hand side of (3.12) converges, and we obtain (3.11). 
Proof of Theorem 2.3 We provide calculations for the more involved case $F_{1}$. For $F$ the calculations become simpler as they do not have to take care of a potential singularity at $x=0$. For $\lambda \in \mathbb{C}$ with $\operatorname{Re} \lambda>0$ we use (3.5) to obtain

$$
\begin{aligned}
\|R(\lambda) f\|_{1} \leq & \int_{0}^{\infty} \frac{|f(x)|}{\left|\lambda+x^{\alpha}\right|}(1+x) \mathrm{d} x \\
& +(2+v) \int_{0}^{\infty}\left(\frac{(1+x) x^{\nu}}{\left|\lambda+x^{\alpha}\right|^{\omega+1}} \int_{x}^{\infty}\left|\lambda+s^{\alpha}\right|^{\omega-1} s^{\alpha-\nu-1}|f(s)| \mathrm{d} s\right) \mathrm{d} x \\
= & : I_{1}+(2+v) I_{2} .
\end{aligned}
$$

Since $\operatorname{Re} \lambda>0$, we have $\left|\lambda+x^{\alpha}\right| \geq|\lambda|$ for $x \geq 0$, and hence $I_{1} \leq\|f\|_{1} /|\lambda|$. To estimate $I_{2}$, we consider two cases: $\alpha \geq v+2$ and $0<\alpha<v+2$. In the former case we have $\omega-1 \leq 0$ and therefore

$\left|\lambda+s^{\alpha}\right|^{\omega-1} s^{\alpha-\nu-1} \leq s^{\alpha(\omega-1)} s^{\alpha-\nu-1}=s$,

which implies that

$$
\begin{aligned}
I_{2} & =\int_{0}^{\infty}\left(\left|\lambda+s^{\alpha}\right|^{\omega-1} s^{\alpha-\nu-1}|f(s)| \int_{0}^{s} \frac{(1+x) x^{\nu}}{\left|\lambda+x^{\alpha}\right|^{\omega+1}} \mathrm{~d} x\right) \mathrm{d} s \\
& \leq \int_{0}^{\infty}\left(s|f(s)| \int_{0}^{s} \frac{x^{\nu}+x^{\nu+1}}{\left|\lambda+x^{\alpha}\right|^{\omega+1}} \mathrm{~d} x\right) \mathrm{d} s \leq\left(\frac{M_{\alpha, \nu, v}}{|\lambda|^{1+\frac{1}{\alpha}}}+\frac{M_{\alpha, \nu, \nu+1}}{|\lambda|}\right) \int_{0}^{\infty} s|f(s)| \mathrm{d} s,
\end{aligned}
$$

where we applied Lemma 3.3(b) twice. If $\operatorname{Re} \lambda \geq 1$, then the latter expression is bounded from above by

$$
\frac{M_{\alpha, v, v}+M_{\alpha, \nu, v+1}}{|\lambda|}\|f\|_{1} \text {. }
$$

In the second case, $0<\alpha<v+2$, we have $\omega-1>0$. On applying Lemma 3.2 we obtain, with $C=C_{\omega-1}^{\prime}$,

$$
\begin{aligned}
I_{2}= & \int_{0}^{\infty}\left(\left|\lambda+s^{\alpha}\right|^{\omega-1} s^{\alpha-\nu-1}|f(s)| \int_{0}^{s} \frac{(1+x) x^{\nu}}{\left|\lambda+x^{\alpha}\right|^{\omega+1}} \mathrm{~d} x\right) \mathrm{d} s \\
\leq & C \int_{0}^{\infty}\left(\left(|\lambda|^{\omega-1}+s^{-\alpha+\nu+2}\right) s^{\alpha-\nu-1}|f(s)| \int_{0}^{s} \frac{(1+x) x^{\nu}}{\left|\lambda+x^{\alpha}\right|^{\omega+1}} \mathrm{~d} x\right) \mathrm{d} s \\
= & C \int_{0}^{\infty}\left(|\lambda|^{\omega-1} s^{\alpha-\nu-1}|f(s)| \int_{0}^{s} \frac{x^{\nu}}{\left|\lambda+x^{\alpha}\right|^{\omega+1}} \mathrm{~d} x\right) \mathrm{d} s \\
& +C \int_{0}^{\infty}\left(|\lambda|^{\omega-1} s^{\alpha-\nu-1}|f(s)| \int_{0}^{s} \frac{x^{\nu+1}}{\left|\lambda+x^{\alpha}\right|^{\omega+1}} \mathrm{~d} x\right) \mathrm{d} s \\
& +C \int_{0}^{\infty}\left(s|f(s)| \int_{0}^{s} \frac{(1+x) x^{\nu}}{\left|\lambda+x^{\alpha}\right|^{1+\omega}} \mathrm{d} x\right) \mathrm{d} s=: C\left(I_{3}+I_{4}+I_{5}\right) .
\end{aligned}
$$

The last integral, $I_{5}$, can be estimated as in the previous case (in the last step we have not used the assumption that $\alpha \geq 2+v$ ). 
For the second integral, $I_{4}$, we can use $\left|\lambda+x^{\alpha}\right|^{\omega+1}=\left|\lambda+x^{\alpha}\right|^{\omega}\left|\lambda+x^{\alpha}\right| \geq|\lambda|^{\omega} x^{\alpha}$ to obtain

$$
\begin{aligned}
I_{4} & =\int_{0}^{\infty}\left(|\lambda|^{\omega-1} s^{\alpha-\nu-1}|f(s)| \int_{0}^{s} \frac{x^{\nu+1}}{\left|\lambda+x^{\alpha}\right|^{\omega+1}} \mathrm{~d} x\right) \mathrm{d} s \\
& \leq \int_{0}^{\infty}\left(|\lambda|^{\omega-1} s^{\alpha-\nu-1}|f(s)| \int_{0}^{s} \frac{x^{\nu+1}}{|\lambda|^{\omega} x^{\alpha}} \mathrm{d} x\right) \mathrm{d} s \\
& =\frac{1}{(v+2-\alpha)|\lambda|} \int_{0}^{\infty} s|f(s)| \mathrm{d} s \leq \frac{1}{(v+2-\alpha)|\lambda|}\|f\|_{1} .
\end{aligned}
$$

On applying Lemma 3.3(a) we obtain the following estimate for the integral $I_{3}$ :

$$
\begin{aligned}
I_{3} & =\int_{0}^{\infty}\left(|\lambda|^{\omega-1} s^{\alpha-\nu-1}|f(s)| \int_{0}^{s} \frac{x^{\nu}}{\left|\lambda+x^{\alpha}\right|^{\omega+1}} \mathrm{~d} x\right) \mathrm{d} s \\
& \leq \int_{0}^{\infty}\left(|\lambda|^{\omega-1} s^{\alpha-\nu-1}|f(s)| \frac{C_{\alpha, \nu}}{|\lambda|^{1+\frac{1}{\alpha}}} \int_{0}^{s /|\lambda|^{1 / \alpha}} \frac{t^{\nu}}{\left(1+t^{\alpha}\right)^{\omega+1}} \mathrm{~d} t\right) \mathrm{d} s \\
& =\frac{C_{\alpha, \nu}}{|\lambda|^{2-\frac{v+1}{\alpha}}} \int_{0}^{\infty}\left(s^{\alpha-\nu-1}|f(s)| \int_{0}^{s /|\lambda|^{1 / \alpha}} \frac{t^{\nu}}{\left(1+t^{\alpha}\right)^{\omega+1}} \mathrm{~d} t\right) \mathrm{d} s=: C_{\alpha, \nu} I_{3}^{\prime} .
\end{aligned}
$$

Let us consider two subcases: $0<\alpha \leq v+1$ and $v+1<\alpha<v+2$. In the first case we have, in particular, $\alpha \leq 1$ and hence $s^{\alpha} \leq 1+s$, which yields

$$
\begin{aligned}
I_{3}^{\prime} & \leq \frac{1}{|\lambda|^{2-\frac{v+1}{\alpha}}} \int_{0}^{\infty}\left(s^{\alpha-v-1}|f(s)| \int_{0}^{s /|\lambda|^{1 / \alpha}} t^{\nu} \mathrm{d} t\right) \mathrm{d} s \\
& =\frac{1}{(v+1)|\lambda|^{2}} \int_{0}^{\infty} s^{\alpha}|f(s)| \mathrm{d} s \leq \frac{1}{(\nu+1)|\lambda|^{2}} \int_{0}^{\infty}(1+s)|f(s)| \mathrm{d} s=\frac{1}{(v+1)|\lambda|^{2}}\|f\|_{1},
\end{aligned}
$$

which for $\operatorname{Re} \lambda \geq 1$ is bounded by $\|f\|_{1} /((v+1)|\lambda|)$. In the second subcase, $v+1<\alpha<v+2$, we see that $2-(v+1) / \alpha>1$ and, again for $\operatorname{Re} \lambda \geq 1$, we obtain

$$
\begin{aligned}
I_{3}^{\prime} & \leq \frac{1}{|\lambda|} \int_{0}^{\infty}\left(s^{\alpha-\nu-1}|f(s)| \int_{0}^{s /|\lambda|^{1 / \alpha}} \frac{t^{\nu}}{\left(1+t^{\alpha}\right)^{\omega+1}} \mathrm{~d} t\right) \mathrm{d} s \\
& \leq \frac{1}{|\lambda|} \int_{0}^{\infty}\left(s^{\alpha-\nu-1}|f(s)| \int_{0}^{\infty} \frac{t^{\nu}}{\left(1+t^{\alpha}\right)^{\omega+1}} \mathrm{~d} t\right) \mathrm{d} s=\frac{C^{\prime}}{|\lambda|} \int_{0}^{\infty} s^{\alpha-\nu-1}|f(s)| \mathrm{d} s \leq \frac{C^{\prime}}{|\lambda|}\|f(s)\|_{1},
\end{aligned}
$$

where the inner integral in the second line is finite on account of $\alpha+\alpha \omega-v=\alpha+2>1$, and the last estimate follows from $0 \leq \alpha-v-1 \leq 1$, which holds by $v+1<\alpha<v+2$.

Putting all estimates together, we see that there is a constant $M$ such that, for all $\lambda \in \mathbb{C}$ with $\operatorname{Re} \lambda \geq 1$, we have $\|R(\lambda) f\|_{1} \leq \frac{M}{|\lambda|}\|f\|_{1}$,

where $R(\lambda)$ is the resolvent of $F_{1}$. Hence it follows from [20, Proposition 2.1.11] that $F_{1}$ is sectorial, which implies that $\left(S_{F_{1}}(t)\right)_{t \geq 0}$ is analytic. 


\section{Intermediate spaces associated with $F_{m}$}

We know that $D\left(A_{1}\right)=X_{1+\alpha} \subset D\left(F_{1}\right)$ and that, in general, the inclusion is strict; see the paragraph after Proposition 6.1 and [9, Remark 8.16]. However, we can prove the following lemma.

Lemma 4.1 For any $0 \leq \gamma<\alpha$ we have

$D\left(F_{1}\right) \subset X_{1+\gamma}$.

Proof We prove that $R(\lambda) f \in X_{1+\gamma}$ for all $f \in X_{1}$ and some positive $\lambda$. The first term in the representation (3.5) is clearly in $X_{1+\gamma}$. Hence it is sufficient to show that the following integral is finite:

$$
\begin{aligned}
\int_{0}^{\infty} & \left(\frac{\left(1+x^{1+\gamma}\right) x^{\nu}}{\left(\lambda+x^{\alpha}\right)^{\omega+1}} \int_{x}^{\infty}\left(\lambda+s^{\alpha}\right)^{\omega-1} s^{\alpha-\nu-1}|f(s)| \mathrm{d} s\right) \mathrm{d} x \\
\quad= & \int_{0}^{\infty}|f(s)|(1+s)\left(\left(\lambda+s^{\alpha}\right)^{\omega-1} \frac{s^{\alpha-\nu-1}}{1+s} \int_{0}^{s} \frac{\left(1+x^{1+\gamma}\right) x^{\nu}}{\left(\lambda+x^{\alpha}\right)^{\omega+1}} \mathrm{~d} x\right) \mathrm{d} s=: \int_{0}^{\infty}|f(s)|(1+s) \Phi(s) \mathrm{d} s .
\end{aligned}
$$

Now, the inner integrand behaves like $x^{\nu}$ for small $x$ and like $x^{1+\gamma+\nu-\alpha-\nu-2}=x^{-1+\gamma-\alpha}$ for large $x$, and thus the integral is $\mathrm{O}\left(s^{\nu+1}\right)$ as $s \rightarrow 0$ and bounded as $s \rightarrow \infty$ provided that $\gamma<\alpha$. Hence $\Phi(s)=\mathrm{O}\left(s^{\alpha}\right)$ as $s \rightarrow 0$ and $\Phi(s)=\mathrm{O}(1)$ as $s \rightarrow \infty$, which shows that $R(\lambda) f \in X_{1+\gamma}$.

Since $\left(F_{1}, D\left(F_{1}\right)\right)$ is the infinitesimal generator of the analytic semigroup $\left(S_{F_{1}}(t)\right)_{t \geq 0}$, we can define intermediate spaces associated with $F_{1}$; see [20, Section 2.2]. As it is more convenient to deal with an invertible generator, we examine the operator $F_{1, r}$ defined by

$F_{1, r}:=F_{1}-r I, \quad D\left(F_{1, r}\right)=D\left(F_{1}\right)$,

where $r>0$ is a positive constant, large enough for $0 \in \rho\left(F_{1, r}\right)$. The abstract Cauchy problem associated with the fragmentation equation then takes the form

$u_{t}=r u+F_{1, r} u, \quad u(0) \in D\left(F_{1}\right)$.

The operator $\left(F_{1, r},\left(D\left(F_{1}\right)\right)\right.$ generates a bounded analytic semigroup $\left(S_{F_{1, r}}(t)\right)_{t \geq 0}$ where $S_{F_{1, r}}(t)=\mathrm{e}^{-r t} S_{F_{1}}(t)$ for each $t \geq 0$. Moreover, the fact that $F_{1, r}$ is invertible means that the norm $\|f\|_{1, F}:=\left\|F_{1, r} f\right\|_{1}, f \in D\left(F_{1}\right)$ is equivalent to the graph norm on $D\left(F_{1}\right)$.

If $Y \subset X$ are Banach spaces with $Y$ continuously embedded in $X$, then one can construct real interpolation spaces $(X, Y)_{\theta, p}, 0<\theta \leq 1,1 \leq p \leq \infty$, by means of the interpolating function

$K(t, x, X, Y):=\inf _{\substack{x=a+b \\ a \in X, b \in Y}}\left(\|a\|_{X}+t\|b\|_{Y}\right)$,

namely

$$
\begin{aligned}
& (X, Y)_{\theta, p}:=\left\{x \in X: t \rightarrow t^{-\theta-1 / p} K(t, x, X, Y) \in L^{p}(0,1)\right\}, \\
& \|x\|_{(X, Y)_{\theta, p}}:=\left\|t^{-\theta-1 / p} K(t, x, X, Y)\right\|_{L^{p}(0,1)} .
\end{aligned}
$$

Since $X_{1+\alpha} \subset D\left(F_{1, r}\right) \subset X_{1+\gamma}, \gamma<\alpha$, with continuous injections, it follows from the definition of the interpolation space that

$\left(X_{1}, X_{1+\alpha}\right)_{\theta, p} \subset\left(X_{1}, D\left(F_{1, r}\right)\right)_{\theta, p} \subset\left(X_{1}, X_{1+\gamma}\right)_{\theta, p}, \quad 0<\theta \leq 1,1 \leq p \leq \infty$,

with continuous injections. We shall focus on the case $p=1$. Since in this case the interpolation space is trivial for $\theta=1$, we exclude $\theta=1$ in the following; see [20, p. 16]. Using [30, Theorem 1.18.5] we find that $\left(X_{1}, X_{1+\delta}\right)_{\theta, 1}=X_{1+\theta \delta}$ for any $\delta \geq 0$ and hence

$X_{1+\theta \alpha} \hookrightarrow\left(X_{1}, D\left(F_{1, r}\right)\right)_{\theta, 1} \hookrightarrow X_{1+\theta \gamma}, \quad 0<\theta<1$. 
The above inclusions can be linked with the intermediate spaces of $F_{1, r}$, which are useful for the investigation of the dynamics of (1.3). Following [20, Section 2.2.1] we define

$$
\begin{aligned}
& D_{F_{1, r}}(\theta, 1):=\left\{x \in X: t \rightarrow v(t):=\left\|t^{-\theta} F_{1, r} S_{F_{1, r}}(t) x\right\|_{X_{1}} \in L^{1}(0,1)\right\}, \\
& \|x\|_{D_{F_{1, r}}(\theta, 1)}:=\|x\|_{X_{1}}+\|v(t)\|_{L^{1}(0,1)} .
\end{aligned}
$$

It follows from [20, Proposition 2.2.2] that

$D_{F_{1, r}}(\theta, 1)=\left(X_{1}, D\left(F_{1, r}\right)\right)_{\theta, 1}$,

which, by (4.4), implies that

$X_{1+\theta \alpha} \hookrightarrow D_{F_{1, r}}(\theta, 1) \hookrightarrow X_{1+\theta \gamma}, \quad 0<\theta<1$,

provided that $\gamma<\alpha$.

\section{Proof of Theorem 2.4}

As a prelude to presenting the calculations that establish our main result, namely Theorem 2.4, let us make precise the connection that exists between operators which in essence take the same form but are defined in different $X_{m}$ spaces. In particular, we observe that $F_{m}=-A_{m}+B_{m}$ defined on $D\left(A_{m}\right)$ and acting in $X_{m}, m>1$, is a restriction of $\left(F_{1}, D\left(F_{1}\right)\right)$ (as the latter is the closure of $\left(-A_{1}+B_{1}, D\left(A_{1}\right)\right)$ ). Since both $R\left(\lambda, F_{1}\right)$ and $R\left(\lambda, F_{m}\right)$ exist for large $\lambda$, we have $R\left(\lambda, F_{m}\right)=\left.R\left(\lambda, F_{1}\right)\right|_{X_{m}}$. It follows that $S_{F_{m}}(t)=\left.S_{F_{1}}(t)\right|_{X_{m}}$, and the same is also true for other semigroups constructed in this section.

Proof of Theorem 2.4 Let the coagulation kernel satisfy (1.5) with $0 \leq \sigma \leq \rho<\alpha$. If we fix any $\theta \in(\rho / \alpha, 1)$, then $\theta \gamma=\rho$ for some $\gamma \in(\rho, \alpha)$ and therefore, by (4.6), $D_{F_{1, r}}(\theta, 1) \hookrightarrow X_{1+\rho}$. We consider the version of (1.3) that takes into account the definition of $F_{1, r}$, namely

$$
\begin{aligned}
\partial_{t} u(x, t)= & -\left(x^{\alpha}+r\right) u(x, t)+\int_{x}^{\infty} a(y) b(x \mid y) u(y, t) \mathrm{d} y \\
& \quad+r u(x, t)-u(x, t) \int_{0}^{\infty} k(x, y) u(y, t) \mathrm{d} y+\frac{1}{2} \int_{0}^{x} k(x-y, y) u(x-y, t) u(y, t) \mathrm{d} y \\
=: & {\left[F_{1, r} u\right](x, t)+\left[C_{1, r} u\right](x, t) . }
\end{aligned}
$$

On using the inequalities $\left(1+x^{\rho}\right)(1+x) \leq C\left(1+x^{\rho+1}\right)$ and $1+x^{\sigma} \leq 2\left(1+x^{\rho}\right)$ with some $C>0$ and the continuous embedding of $D_{F_{1, r}}(\theta, 1)$ in $X_{1+\rho}$, we obtain, for $f, g \in D_{F_{1, r}}(\theta, 1)$,

$$
\begin{aligned}
& \int_{0}^{\infty}|f(x)|\left(\int_{0}^{\infty} k(x, y)|g(y)| \mathrm{d} y\right)(1+x) \mathrm{d} x \\
& \leq K \int_{0}^{\infty} \int_{0}^{\infty}\left(\left(1+x^{\rho}\right)\left(1+y^{\sigma}\right)+\left(1+x^{\sigma}\right)\left(1+y^{\rho}\right)\right)|f(x)||g(y)|(1+x) \mathrm{d} x \mathrm{~d} y \\
& \leq K_{1}\left(\|f\|_{1+\rho}\|g\|_{\sigma}+\left\|\left.f\right|_{1+\sigma}\right\| g \|_{\rho}\right) \leq K_{2}\|f\|_{1+\rho}\|g\|_{1+\rho} \\
& \leq K_{3}\|f\|_{D_{F_{1, r}}(\theta, 1)}\|g\|_{D_{F_{1, r}}}(\theta, 1)
\end{aligned}
$$

and, likewise, 


$$
\begin{aligned}
& \int_{0}^{\infty}\left(\int_{0}^{x} k(x-y, y)|f(y)||g(x-y)| \mathrm{d} y\right)(1+x) \mathrm{d} x \\
& \quad=\int_{0}^{\infty} \int_{0}^{\infty} k(x, y)|f(y)||g(x)|(1+x+y) \mathrm{d} x \mathrm{~d} y \\
& \quad \leq K \int_{0}^{\infty} \int_{0}^{\infty}|f(y)||g(x)|\left(\left(1+x^{\rho}\right)\left(1+y^{\sigma}\right)+\left(1+y^{\rho}\right)\left(1+x^{\sigma}\right)\right)((1+x)+(1+y)) \mathrm{d} y \mathrm{~d} x \\
& \quad \leq K_{4}\|f\|_{1+\rho}\|g\|_{1+\rho} \leq K_{5}\|f\|_{D_{F_{1, r}}}(\theta, 1)\|g\|_{D_{F_{1, r}}(\theta, 1)} .
\end{aligned}
$$

If we now investigate the Lipschitz continuity of $C_{1, r}$, then we observe that, whenever $f, g \in D_{F_{1, r}}(\theta, 1)$, the linear component satisfies $\|r f-r g\|_{1} \leq r\|f-g\|_{D_{F_{1, r}}(\theta, 1)}$. Since, for a generic quadratic operator $\mathrm{Q}$ generated by a bilinear operator $\mathrm{B}$, we have

$\mathrm{Q}(u)-\mathrm{Q}(v)=\mathrm{B}(u, u)-\mathrm{B}(v, v)=\mathrm{B}(u, u-v)+\mathrm{B}(u-v, v)$,

we obtain from (5.3) and (5.4) that

$\left\|C_{1, r} f-C_{1, r} g\right\|_{1} \leq\left(r+\left(K_{3}+K_{5}\right)\left(\|f\|_{D_{F_{1, r}}(\theta, 1)}+\|g\|_{D_{F_{1, r}}(\theta, 1)}\right)\right)\|f-g\|_{D_{F_{1, r}}(\theta, 1)}$.

Thus we see that the assumptions of [20, Theorem 7.1.2] (with $X_{\alpha}$ of op. cit. equal to $D_{F_{1, r}}(\theta, 1)$ ) and, for regularity, of [20, Proposition 7.1.10] are satisfied. Therefore, for any $\stackrel{\bullet}{u} \in D_{F_{1, r}}(\theta, 1)$, there is a unique mild solution $u$ to (2.4) in $D_{F_{1, r}}(\theta, 1)$ which, moreover, satisfies

$u \in C\left([0, \tau), D_{F_{1, r}}\left(\theta^{\prime}, 1\right)\right) \cap C^{1}\left((0, \tau), X_{1}\right) \cap C\left((0, \tau), D\left(F_{1}\right)\right)$

for $\theta^{\prime} \in(\rho / \alpha, \theta)$. To complete the proof of the local existence part of Theorem 2.4, we again use (4.6) to deduce that $D_{F_{1, r}}\left(\theta^{\prime}, 1\right) \hookrightarrow X_{1+\left(\rho / \theta^{\prime}\right) \theta^{\prime}}=X_{1+\rho}$ and, when $\rho<\beta<\alpha, X_{1+\beta}=X_{1+(\beta / \alpha) \alpha} \hookrightarrow D_{F_{1, r}}(\theta, 1) \hookrightarrow X_{1+\rho}$. Note that to maximise the set of initial conditions for which the problem is locally solvable we should choose $\beta$ as close to $\rho$ as possible due to the fact that $X_{1+\beta_{2}} \hookrightarrow X_{1+\beta_{1}}$ when $\beta_{1} \leq \beta_{2}$. Moreover, when $\beta \geq \alpha$, the inclusion $X_{1+\beta} \hookrightarrow D_{F_{1, r}}(\theta, 1)$ is automatically satisfied.

Turning now to the proof of global existence, it is clear from (4.6) that $D_{F_{1, r}}(\theta, 1) \subset X_{1}$. From [20, Theorem 7.1.2] we know that the local solutions depend continuously on their initial conditions. More precisely, denote by $\phi\left(t, f_{1}\right), \phi\left(t, f_{2}\right)$ the local solutions emanating from $f_{1}, f_{2}$. Then, for any $\bar{f} \in D_{F_{1, r}}(\theta, 1)$ there are $\varepsilon, M>0$ and $\tau>0$ such that, if $\left\|f_{i}-\bar{f}\right\|_{D_{F_{1, r}}(\theta, 1)}<\varepsilon, i=1,2$, then

$\left\|\phi\left(t, f_{1}\right)-\phi\left(t, f_{2}\right)\right\|_{D_{F_{1, r}}(\theta, 1)} \leq M\left\|f_{1}-f_{2}\right\|_{D_{F_{1, r}}(\theta, 1)}, \quad t \in(0, \tau)$.

Due to the considerations above we can write

$\left\|\phi\left(t, f_{1}\right)-\phi\left(t, f_{2}\right)\right\|_{1} \leq\left\|\phi\left(t, f_{1}\right)-\phi\left(t, f_{2}\right)\right\|_{D_{F_{1, r}}(\theta, 1)} \leq M\left\|f_{1}-f_{2}\right\|_{D_{F_{1, r}}(\theta, 1)} \leq M_{1}\left\|f_{1}-f_{2}\right\|_{1+\beta}$.

Consider now $0 \leq \stackrel{\circ}{u} \in X_{1+\beta}$. This initial value can be approximated in $X_{1+\beta}$ by a non-negative sequence $f_{n} \in X_{m+\rho}$. Note that $\tau$ in (5.6) is the same for all approximating initial conditions chosen from the ball of radius $\varepsilon$ specified above, and thus all approximating solutions are defined at least on $[0, \tau)$. Since, by Theorem 2.2, $\phi\left(t, f_{n}\right)$ are non-negative functions, $\phi(t, \stackrel{\circ}{u})$ is also non-negative on $(0, \tau)$. Hence we have the existence of local non-negative solutions. Now, as (5.6) is based on the Gronwall lemma, it holds on every finite interval of joint existence. Thus, if additionally (1.6) holds with $\rho \leq 1$, then extending $\phi(t, \stackrel{\circ}{u})$ to its maximal interval of existence and using the fact that approximating solutions $\phi\left(t, f_{n}\right)$ exist for all $t$, we establish that $\phi(t, \stackrel{\bullet}{u})$ also exists globally in time. 


\section{6 'Spurious' solutions to continuous fragmentation equations}

In this section we consider the fragmentation part of (1.3) in the particular case when $\alpha=1$ and $v=0$, namely

$\partial_{t} u(x, t)=-x u(x, t)+2 \int_{x}^{\infty} u(y, t) \mathrm{d} y$.

We consider the process only in $Y$. It follows from (3.5) that the resolvent of $F$ is given by

$[R(\lambda) u](x)=\frac{u(x)}{\lambda+x}+\frac{2}{(\lambda+x)^{3}} \int_{x}^{\infty}(\lambda+s) u(s) \mathrm{d} s$.

In this case, it is possible to obtain a complete characterisation of $D(F)$.

Proposition 6.1 The domain of the operator $F$ is given by

$D(F)=\left\{f \in Y: \mathcal{F} f \in Y, \lim _{x \rightarrow \infty}\left(x^{2} \int_{x}^{\infty} f(s) \mathrm{d} s\right)=0\right\}$.

Proof Let $f \in D(F)$. Then, for some $\lambda>0$, there exists $g \in Y$ such that $f=R(\lambda) g$. It is easy to see that $\mathcal{F} f=\mathcal{F} R(\lambda) g=\lambda f-g \in Y$. Moreover, for $x>0$,

$$
\begin{aligned}
x^{2} \int_{x}^{\infty} f(s) \mathrm{d} s & =x^{2} \int_{x}^{\infty}[R(\lambda) g](s) \mathrm{d} s=x^{2} \int_{x}^{\infty}\left(\frac{g(s)}{\lambda+s}+\frac{2}{(\lambda+s)^{3}} \int_{s}^{\infty}(\lambda+r) g(r) \mathrm{d} r\right) \mathrm{d} s \\
& =x^{2} \int_{x}^{\infty} \frac{g(s)}{\lambda+s} \mathrm{~d} s+x^{2} \int_{x}^{\infty}\left(\int_{x}^{r} \frac{2}{(\lambda+s)^{3}} \mathrm{~d} s\right)(\lambda+r) g(r) \mathrm{d} r \\
& =x^{2} \int_{x}^{\infty} \frac{g(s)}{\lambda+s} \mathrm{~d} s+x^{2} \int_{x}^{\infty}\left(\frac{1}{(\lambda+x)^{2}}-\frac{1}{(\lambda+r)^{2}}\right)(\lambda+r) g(r) \mathrm{d} r=\frac{x^{2}}{(\lambda+x)^{2}} \int_{x}^{\infty}(\lambda+r) g(r) \mathrm{d} r,
\end{aligned}
$$

which converges to 0 as $x \rightarrow \infty$. Hence $f$ is in the set on the right-hand side of (6.3).

Conversely, assume that $f$ is in the set on the right-hand side of (6.3). Then, for $\lambda>0$, we can define $g:=$ $(\lambda-\mathcal{F}) f \in Y$ and, for $x>0$, we obtain

$$
\begin{aligned}
{[R(\lambda) g](x) } & =[R(\lambda)(\lambda-\mathcal{F}) f](x) \\
& =\frac{1}{\lambda+x}\left((\lambda+x) f(x)-2 \int_{x}^{\infty} f(s) \mathrm{d} s\right)+\frac{2}{(\lambda+x)^{3}} \int_{x}^{\infty}(\lambda+s)\left((\lambda+s) f(s)-2 \int_{s}^{\infty} f(r) \mathrm{d} r\right) \mathrm{d} s \\
& =f(x)-\frac{2}{(\lambda+x)^{3}} \int_{x}^{\infty}\left((\lambda+x)^{2} f(s)-(\lambda+s)^{2} f(s)+2(\lambda+s) \int_{s}^{\infty} f(r) \mathrm{d} r\right) \mathrm{d} s \\
& =f(x)-\frac{2}{(\lambda+x)^{3}}\left[(\lambda+x)^{2} \int_{x}^{\infty} f(s) \mathrm{d} s+\int_{x}^{\infty} \frac{\partial}{\partial s}\left((\lambda+s)^{2} \int_{s}^{\infty} f(r) \mathrm{d} r\right) \mathrm{d} s\right] \\
& =f(x)-\frac{2}{(\lambda+x)^{3}}\left[(\lambda+x)^{2} \int_{x}^{\infty} f(s) \mathrm{d} s+\lim _{b \rightarrow \infty}\left((\lambda+b)^{2} \int_{b}^{\infty} f(r) \mathrm{d} r\right)-(\lambda+x)^{2} \int_{x}^{\infty} f(r) \mathrm{d} r\right] \\
& =f(x) .
\end{aligned}
$$

Hence $f=R(\lambda) g$, which implies that $f \in D(F)$. 
Let $F_{\max }$ be the maximal operator associated with $\mathcal{F}$, i.e. $D\left(F_{\max }\right)=\{f \in Y: \mathcal{F} f \in Y\}, F_{\max } f=\mathcal{F} f$ for $f \in D\left(F_{\max }\right)$. With $m>1$, there is strict inclusion everywhere in the following chain

$D\left(A_{m}\right) \subset D\left(A_{1}\right) \subset D(F) \subset D\left(F_{\max }\right)$;

this can be seen with the following functions:

$f_{1}(x)=\frac{1}{(\lambda+x)^{3}}, \quad f_{2}(x)=\frac{1}{(\lambda+x)^{3} \ln (\lambda+x)}, \quad f_{3}(x)=\frac{1}{(\lambda+x)^{3}(\ln (\lambda+x))^{\alpha}}$,

where $\lambda>0$ for $f_{1}$ and $\lambda>1$ for $f_{2}, f_{3}$ and $\alpha>1$, which satisfy

$f_{1} \in D\left(F_{\max }\right) \backslash D(F), \quad f_{2} \in D(F) \backslash D\left(A_{1}\right), \quad f_{3} \in D\left(A_{1}\right) \backslash D\left(A_{m}\right)$

for $m>1$.

If one considers the evolution equation

$u_{t}=F_{\max } u$

instead of $u_{t}=F u$, then one loses uniqueness, as the following example shows: for $\lambda>0$ set

$u(x, t):=\mathrm{e}^{\lambda t} f_{1}(x)-\left[S_{F}(t) f_{1}\right](x), \quad t \geq 0, x \geq 0$.

It is easy to see that $u(\cdot, t) \in D\left(F_{\max }\right)$ for all $t \geq 0$ and that the function $t \mapsto u(\cdot, t)$ is continuous on $[0, \infty)$, strongly differentiable on $(0, \infty)$ and satisfies (6.4) and the initial condition $u(x, 0)=0$. Clearly, the function $u$ is non-trivial since $u(\cdot, t) \notin D(F)$ for $t>0$; note that $S_{F}(t) f_{1} \in D(F)$ for $t>0$. This phenomenon was analysed in [31].

To conclude, we now consider the specific case when $u$ is given by

$u(x, t)=t^{2} \mathrm{e}^{-x t}$.

Routine calculations show that this function is a pointwise solution to (6.1), and satisfies the relations $\int_{0}^{\infty} x|u(x, t)| \mathrm{d} x$ $<\infty$ and $\int_{0}^{\infty} x^{2}|u(x, t)| \mathrm{d} x<\infty$ for all $t>0$, and therefore $u(\cdot, t) \in D(F)$. The function $t \mapsto u(\cdot, t)$ is strongly differentiable on $(0, \infty)$, i.e.

$\int_{0}^{\infty} x\left|\frac{1}{h}(u(x, t+h)-u(x, t))-\left(2 t \mathrm{e}^{-x t}-t^{2} x \mathrm{e}^{-x t}\right)\right| \mathrm{d} x \rightarrow 0 \quad$ as $h \rightarrow 0$

for all $t>0$, which can be shown using Lebesgue's dominated convergence theorem. Thus, $u_{t}=F u, t>0$, in $Y$. Clearly, $u(x, 0)=0$ for all $x \in \mathbb{R}_{+}$, so we have a non-trivial classical solution of the fragmentation equation (6.1) emanating from zero. This shows that (6.1) is not well posed in the pointwise sense. However, there is no contradiction to the well-posedness of the corresponding abstract Cauchy problem, which is guaranteed by Theorem 2.3, because $u(\cdot, t)$ does not converge in $Y$ as $t \rightarrow 0^{+}$.

To explain the behaviour of $u(\cdot, t)$ as $t \rightarrow 0^{+}$, let us recall the concept of Sobolev towers; see, e.g. [32, Sect. II.5.a]. The first element of this tower is the Sobolev space of order -1 associated with $F$, denoted by $Y_{-1}$ and defined as the completion of $Y$ with respect to the norm

$\|\mathbf{x}\|_{Y_{-1}}=\|R(\lambda) \mathbf{x}\|_{Y}$

for some fixed $\lambda>0$. The semigroup $\left(S_{F}(t)\right)_{t \geq 0}$ extends, by density, to a strongly continuous semigroup $\left(S_{F,-1}(t)\right)_{t \geq 0}$ on $Y_{-1}$ generated by the operator $F_{-1}$ whose resolvent, $R_{-1}(\lambda)$, is the extension of the resolvent $R(\lambda)$ of $F$. This extended resolvent, $R_{-1}(\lambda)$, maps $Y$ bijectively onto $Y_{-1}$, and hence $D\left(F_{-1}\right)=Y$. It follows from [28, Lemma 4.1] that $u(\cdot, t)$ converges in $Y_{-1}$ to an element $\mathbf{u}$ if and only if the limit

$\stackrel{\circ}{v}:=\lim _{t \rightarrow 0^{+}} \int_{t}^{\infty} \mathrm{e}^{-\lambda s} u(\cdot, s) \mathrm{d} s$

exists in $Y$, and in this case, $R_{-1}(\lambda) \stackrel{\circ}{\mathbf{u}}=\stackrel{\circ}{v}$. 
For the function (6.5) the limit on the right-hand side of (6.6) exists and is given by $\stackrel{v}{v}(x)=\int_{0}^{\infty} \mathrm{e}^{-\lambda s} u(x, s) \mathrm{d} s$ since

$\int_{0}^{\infty} x\left|\int_{0}^{t} \mathrm{e}^{-\lambda s} u(x, s) \mathrm{d} s\right| \mathrm{d} x=\int_{0}^{t}\left(\mathrm{e}^{-\lambda s} \int_{0}^{\infty} x u(x, s) \mathrm{d} x\right) \mathrm{d} s=\int_{0}^{t} \mathrm{e}^{-\lambda s} \mathrm{~d} s \rightarrow 0 \quad$ as $t \rightarrow 0^{+}$.

Explicitly, the limit $\stackrel{i}{\text { is }}$

$\grave{v}(x)=\int_{0}^{\infty} \mathrm{e}^{-\lambda s} u(x, s) \mathrm{d} s=\frac{2}{(\lambda+x)^{3}}$.

For an interpretation of this limit, we consider the mass distribution

$f_{m}(x)= \begin{cases}\frac{2}{2 m-1} & \text { if } m-1 \leq x \leq m, \\ 0 & \text { otherwise, }\end{cases}$

for $m \in \mathbb{N}$, which is a density describing a unit mass ensemble of particles having masses in the interval $[m-1, m]$. Let us choose $\lambda=1$ in the following. Then, with (6.2), we obtain

$\left[R(1) f_{m}\right](x)= \begin{cases}\frac{2(2 m+1)}{(2 m-1)(x+1)^{3}} & \text { if } 0 \leq x \leq m-1, \\ \frac{2(m+1)^{2}}{(2 m-1)(x+1)^{3}} & \text { if } m-1<x \leq m, \\ 0 & \text { otherwise, }\end{cases}$

and

$\begin{aligned} & \int_{0}^{\infty} x \mid {\left[R(1) f_{m}\right](x)-\frac{2}{(1+x)^{3}} \mid \mathrm{d} x } \\ & \quad=\int_{0}^{m-1} \frac{4 x}{(2 m-1)(x+1)^{3}} \mathrm{~d} x+\int_{m-1}^{m} \frac{2\left(m^{2}+2\right) x}{(2 m-1)(x+1)^{3}} \mathrm{~d} x+\int_{m}^{\infty} \frac{2 x}{(1+x)^{3}} \mathrm{~d} x \rightarrow 0\end{aligned}$

as $m \rightarrow \infty$. Hence $\left(f_{m}\right)_{m \geq 1}$ converges in $Y_{-1}$ to the 'singular' initial value $\stackrel{\circ}{\mathbf{u}}$ of $t^{2} \mathrm{e}^{-x t}$. Thus, we can interpret $\stackrel{\circ}{\mathbf{u}}$ as an 'infinite' particle of unit mass.

Finally, the fact that $\left(S_{F}(t)\right)_{t \geq 0}$ is analytic explains why the solution (6.5) is immediately observable for $t>0$, that is, $u(\cdot, t) \in Y$ for $t>0$. Analytic semigroups are immediately smoothing and therefore any solution emanating from an initial condition in any $Y_{-n}$ immediately enters $Y$ (or for that matter, $\bigcap_{k=0}^{\infty} D\left(F^{k}\right)$ ), continuing as a strongly differentiable solution enjoying also a semigroup property

$u\left(\cdot, t_{1}+t_{2}\right)=S_{F}\left(t_{1}\right) u\left(\cdot, t_{2}\right), \quad t_{2}>0, t_{1} \geq 0$.

This example indicates how careful one has to be while looking for explicit solutions of infinite-dimensional dynamical systems such as (6.1) so as not to pick up a spurious solution.

\section{Conclusions}

In recent papers [24,25], we have proved the existence of global classical solutions to the fragmentation-coagulation equation with unbounded fragmentation and coagulation rates. These results required the initial conditions to have finite moments of sufficiently high order. In this paper, we have continued to consider unbounded coagulation rates. By restricting attention to fragmentation rates of power-law type, we have been able to prove that the related 
fragmentation semigroup is analytic in the physically relevant space $Y=L_{1}\left(\mathbb{R}_{+}, x \mathrm{~d} x\right)$. Exploiting this property, we established existence and uniqueness for the full fragmentation-coagulation equation for a large class of initial conditions. The paper is concluded by constructing a solution of a fragmentation equation which has all moments finite for $t>0$ but originates from a non-physical initial condition, which can be interpreted as an 'infinite' particle with unit mass. The existence of such solutions again is possible due to the analyticity of the fragmentation semigroup and hence its smoothing property.

Acknowledgments This research was initiated when the first author visited the University of Strathclyde as a Sir David Anderson Fellow and was continued during his appointment as a Visiting Professor there. The research was also supported by grant N N201605640 of the Polish National Centre for Science.

Open Access This article is distributed under the terms of the Creative Commons Attribution License which permits any use, distribution, and reproduction in any medium, provided the original author(s) and the source are credited.

\section{References}

1. Johansen A, Brauer C, Dullemond CP, Klahr H, Henning T (2008) A coagulation-fragmentation model for the turbulent growth and destruction of preplanetesimals. Astron Astrophys 486:597-613

2. Samsel RW, Perelson AS (1982) Kinetics of roleau formation. Biophys J 37:493-514

3. Vigil RD, Ziff RM (1989) On the stability of coagulation-fragmentation population balances. J Colloid Interface Sci 133:257-264

4. Ziff RM (1980) Kinetics of polymerization. J Stat Phys 23:241-263

5. Amar JG, Popescu MN, Family F (2001) Rate-equation approach to island capture zones and size distributions in epitaxial growth. Phys Rev Lett 86:3092-3095

6. McGrady ED, Ziff RM (1987) "Shattering" transition in fragmentation. Phys Rev Lett 58:892-895

7. McLaughlin DJ, Lamb W, McBride AC (1997) A semigroup approach to fragmentation models. SIAM J Math Anal 28:1158-1172

8. McLaughlin DJ, Lamb W, McBride AC (1997) An existence and uniqueness result for a coagulation and multiple-fragmentation equation. SIAM J Math Anal 28:1173-1190

9. Banasiak J, Arlotti L (2006) Perturbations of positive semigroups with applications. Springer, London

10. Banasiak J, Oukouomi Noutchie SC, Rudnicki R (2009) Global solvability of a fragmentation-coagulation equation with growth and restricted coagulation. J Nonlinear Math Phys 16(1):13-26

11. Stewart IW (1989) A global existence theorem for the global coagulation-fragmentation equation with unbounded kernels. Math Methods Appl Sci 11:627-648

12. Dubovskiı̌ PB, Stewart IW (1996) Existence, uniqueness and mass conservation for the coagulation-fragmentation equation. Math Methods Appl Sci 19:571-591

13. Laurençot $P$ (2000) On a class of continuous coagulation-fragmentation equations. J Differ Equ 167:245-274

14. Giri AK, Kumar J, Warnecke G (2011) The continuous coagulation equation with multiple fragmentation. J Math Anal Appl 374:71-87

15. Banasiak J, Lamb W (2011) Global strict solutions to continuous coagulation-fragmentation equations with strong fragmentation. Proc R Soc Edinb A 141:465-480

16. Aizenman M, Bak TA (1979) Convergence to equilibrium in a system of reacting polymers. Commun Math Phys 65:203-230

17. Banasiak J, Lamb W (2009) Coagulation, fragmentation and growth processes in a size structured population. Discrete Contin Dyn Syst B 11:563-585

18. Banasiak J (2012) Transport processes with coagulation and strong fragmentation. Discrete Contin Dyn Syst B 17:445-472

19. Lamb W (2004) Existence and uniqueness results for the continuous coagulation and fragmentation equation. Math Methods Appl Sci 27:703-721

20. Lunardi A (1995) Analytic semigroups and optimal regularity in parabolic problems. Birkhäuser-Verlag, Basel

21. Aldous DJ (1999) Deterministic and stochastic models for coalescence (aggregation and coagulation): a review of the mean-field theory for probabilists. Bernoulli 5:3-48

22. Smit DJ, Hounslow MJ, Paterson WR (1994) Aggregation and gelation-I. Analytical solutions for CST and batch operation. Chem Eng Sci 49:1025-1035

23. Koch J, Hackbusch W, Sundmacher K (2007) H-matrix methods for linear and quasi-linear integral operators appearing in population balances. Comput Chem Eng 31:745-759

24. Banasiak J (2012) Global classical solutions of coagulation-fragmentation equations with unbounded coagulation rates. Nonlinear Anal Real World Appl 13:91-105

25. Banasiak J, Lamb W (2012) Analytic fragmentation semigroups and continuous coagulation-fragmentation equations with unbounded rates. J Math Anal Appl 391:312-322

26. Banasiak J, Oukoumi Noutchie SC (2010) Controlling number of particles in fragmentation equations. Physica D 239:1422-1435

27. Edwards BF, Cai M, Han H (1990) Rate equation and scaling for fragmentation with mass loss. Phys Rev A 41:5755-5757 
28. Smith L, Lamb W, Langer M, McBride A (2012) Discrete fragmentation with mass loss. J Evol Equ 12:181-201

29. Hardy GH, Littlewood JE, Pólya G (1934) Inequalities. Cambridge University Press, Cambridge

30. Triebel H (1978) Interpolation theory, function spaces, differential operators. North Holland, Amsterdam

31. Banasiak J (2002) On a non-uniqueness in fragmentation models. Math Methods Appl Sci 25:541-556

32. Engel K-J, Nagel R (2000) One-parameter semigroups for linear evolution equations. Springer, New York 